Discussion Paper No. 830

\title{
PRIVATE PROVISION OF PUBLIC GOODS THAT ARE COMPLEMENTS FOR PRIVATE GOODS: APPLICATION TO OPEN SOURCE SOFTWARE DEVELOPMENTS
}

Noriaki Matsushima

Ryusuke Shinohara

February 2012

The Institute of Social and Economic Research Osaka University

6-1 Mihogaoka, Ibaraki, Osaka 567-0047, Japan 


\title{
Private Provision of Public Goods That Are Complements for Private Goods: Application to Open Source Software Developments
}

\author{
Noriaki Matsushima* and Ryusuke Shinohara $^{\dagger}$
}

February 1, 2012

\begin{abstract}
This paper examines the questions of who participates in the provision of a public good through the voluntary participation of agents in the presence of strong complementarity between a public good and a private good. We show that the greater the initial endowment of the private good that agents have, the more likely they are to participate in the provision of the public good. Whether an agent participates does not depend on the efficiency of his/her technology for production of the public good. We extend the basic model and introduce a simple transfer game. We show a sufficient condition so that the voluntary transfer scheme achieves Pareto efficiency.
\end{abstract}

Keywords. Public goods, voluntary participation, strong complementarity, transfer of private goods.

JEL Classification Numbers. C72, H41, L17.

${ }^{*}$ Noriaki Matsushima, Institute of Social and Economic Research, Osaka University, Mihogaoka 61, Ibaraki, Osaka, 567-0047, Japan. Phone: (81)-6-6879-8571. Fax: (81)-6-6879-8583. E-mail: nmatsush@iser.osaka-u.ac.jp

${ }^{\dagger}$ Corresponding author: Ryusuke Shinohara, Faculty of Economics, Shinshu University, 3-1-1 Asahi, Matsumoto, Nagano, 390-8621, Japan. Phone: (81)-263-37-2951. Fax: (81)-263-37-2344. E-mail: ryusukes@shinshu-u.ac.jp 


\section{Introduction}

We examine the provision of a public good for the case in which there is strong complementarity between the public good and a private good. An authentic example of our problem is investment to improve the quality of Open Source Software (OSS), such as Linux and Apache. Because OSS is usually provided free by developers of the software on the web, it is available to everyone at the same quality at no cost. Thus, it can be treated as a public good. OSS also has the nature of a complementary product for private goods used by private agents. Improvement in the quality of Linux, for instance, benefits the users of Linux-based computers. The relation between the qualities of Linux and those of Linux-based computers/applications has the following important property. Highquality computers/applications require Linux to be of similar quality. If the quality of Linux is not sufficient, such computers/applications do not achieve optimum performance. In other words, the quality of Linux functions as the maximum performance capacity of Linux-based computers/applications. That is, the users benefit from high-quality Linuxbased computers/applications only if the quality of Linux is high.

In the presence of strong complementarity between public and private goods, as in Linux and its applications, beneficiaries of the public good are likely to participate voluntarily in provision of the public good. For an individual to obtain substantial utility, it is necessary to consume large quantities of both public and private goods. Thus, when the public good is provided in sufficiently low quantities, an individual voluntarily produces the public good using private goods rather than by free riding. The complementarity between the public and the private goods may result in the participation of many individuals in providing public goods. In fact, there are many projects for OSS; many engineers voluntarily participate in the development of OSS, and many types of high-quality OSS are provided. ${ }^{1}$ This situation concerning OSS seems different from that predicted by the

\footnotetext{
${ }^{1}$ See Lerner and Tirole (2002) for information on the development of OSS.
} 
standard theory of public goods, which states that the free-rider problem causes the underprovision of a public good in comparison with the Pareto-efficient level of supply. In a situation such as OSS development, the following questions arise. Who participates in the public good provision? How is desirable allocation achieved by the many contributors?

To examine these questions, we construct the following theoretical model. There is one public good and one private good. The private good is consumed by agents or is used to provide the public good. Each agent has the technology and the resources (his/her initial endowment of the private good) to provide the public good. The technology and the resources for the public good may differ among agents. To capture the strong complementarity between the private and public goods, all agents are assumed to have the same Leontief utility function with respect to the consumption of the two goods. We examine participation behavior using a voluntary participation game. The participation game consists of two stages. In the first stage, all agents simultaneously choose whether to participate in the provision of a public good. In the second stage, knowing the other agents' participation decisions, the agents who chose to participate simultaneously decide their contribution to a public good, as in a voluntary contribution game (Bergstrom et al., 1987).

We first show that voluntary participation and contribution to the public good at the subgame-perfect Nash equilibrium of the participation game depend solely on an agent's initial endowment of the private good. The greater the initial endowment of the private good that agents have, the more likely they are to participate and contribute to the public good. Becoming a contributor does not depend on the efficiency of their technology for production of the public good: even if an agent has more efficient technology than others, he/she may choose not to contribute. We emphasize that our paper partially supports the recent interesting empirical results of Bitzer and Geishecker (2010), who investigate the question of who actually creates OSS. Using a sample with both contributors and noncontributors to OSS projects, they investigate the view that it is mainly workers with 
high educational attainment that voluntarily contribute to OSS development. They find no positive association whatsoever between formal education and the probability of voluntary OSS contributions. They also find that the length of work hours is highly statistically significant and has a negative impact on the probability of contributing voluntarily to OSS projects. These two findings are related to one of our results: the determinant of contribution to the provision of a public good is not related to the efficiency of agents' contribution but to their initial endowments. Efficiency in our model is related to high educational attainment in their empirical analysis, and initial endowment in our model is negatively related to the length of work hours in their analysis. Based on the similarity between the results in their study and ours, we believe that our model has the potential to explain why someone voluntarily contributes to OSS development.

Second, we examine the Pareto efficiency of the equilibrium allocation in the voluntary participation game. We introduce two criteria of feasibility of allocations, depending upon whether the resource for providing the public good is transferable among agents. The individually feasible allocation is defined such that for each agent, the initial endowment of the private good covers consumption of the private good and contribution to the public good. The socially feasible allocation is defined such that the aggregate initial endowment of the private good in the economy covers the aggregate consumption of the private good and the aggregate contribution to the public good. We show that while the equilibrium allocation is Pareto efficient within the set of individually feasible allocations, it is not necessarily so within the set of socially feasible allocations (we call this $s$-Pareto efficient). We show that the allocation at the subgame-perfect Nash equilibrium of the voluntary participation game is s-Pareto efficient if and only if only the agent with the most efficient production technology is a contributor.

Third, we examine whether agents voluntarily redistribute the private good among them so that the s-Pareto-efficient allocation is achieved. We extend the voluntary participation game so that agents can transfer the private good, based on the model of Jackson 
and Wilkie (2005). We provide a sufficient condition under which voluntary transfer results in s-Pareto efficiency. The sufficient condition implies that contributors expected to produce positive amounts of the public good can improve their utility levels if they can transfer their endowments among them. In the context of OSS, money is considered a transferable resource among engineers. Time can also be such a resource, and is usually nontransferable. There may be a case in which time is transferable through the division of roles among a group of engineers. ${ }^{2}$ Note that the above sufficient condition also implies that the s-Pareto-efficient allocation is not necessarily achieved through voluntary transfer. As an example, we examine the difficulty of achieving s-Pareto-efficient allocation through voluntary transfer.

\section{Related literature}

This paper is closely related to the analysis of the voluntary participation problem in a public good mechanism. Saijo and Yamato (1999) introduce a voluntary participation game and analyze the number of agents voluntarily participating in the public good mechanism when they can freely decide to do so. In Saijo and Yamato's (1999) model, all agents are assumed to have the same Cobb-Douglas utility function. ${ }^{3}$ Healy (2010), Furusawa and Konishi (2011), and Konishi and Shinohara (2012) investigate the voluntary participation problem in other domains of utility functions. The main message of these studies is that if participation in a public good mechanism is not coerced, every agent has an incentive not to participate and to free ride the public good provided by participants. Thus, even if a mechanism is constructed to provide desirable allocations, such as the Pareto-efficient allocation at its equilibrium, it is very difficult to provide all agents with an incentive for voluntary participation. In contrast, Shinohara $(2009,2011)$ points

\footnotetext{
${ }^{2}$ We believe that transfers would also be easy in the following situation. Several agents in a group must perform some duties in cooperation. That is, each agent needs to spend some time performing these duties, spending his/her remaining time consuming the private good or producing the public good. In this context, an agent's initial remaining time is related to the initial endowment. A transfer of one agent's duty to another changes their remaining time.

${ }^{3}$ Saijo and Yamato (2010) extend their analysis to the cases in which agents have different Cobb-Douglas utility functions or a quasilinear utility function.
} 
out that if there is a specific structure for a public good, the Pareto- efficient provision is possible at a subgame-perfect Nash equilibrium in the voluntary participation game. None of these studies has examined how strong complementarity between the private and public goods affects the incentive for agents to participate.

This paper also contributes to the literature on OSS. Academic scholars have investigated the hows and whys of OSS development (Lerner and Tirole, 2002; Johnson, 2002; Franke and von Hippel, 2003; Lakhani and von Hippel, 2003; von Hippel and von Krogh, 2003; Bitzer and Schröder, 2005; Bitzer et al., 2007). In the existing literature, while many empirical analyses have been conducted, there are only a few theoretical investigations using models of public good provision. Johnson (2002) investigates why developers voluntarily provide OSS and how the voluntary development of OSS affects social welfare. Basically, in his model, whether a developer voluntarily contributes to an OSS depends on the relationship between the cost and benefit of development. Unlike us, he does not explicitly model the strong complementarity between the private and public good, which can be observed in many types of OSS. The results are quite different in the sense that while Johnson (2002) explains the incentive for voluntary development according to the benefit from the OSS and its development cost, we explain incentive by the amount of resources held by a developer for developing an OSS. ${ }^{4}$ In this sense, our result is unique.

The remainder of this paper is organized as follows. Section 2 presents the basic model. Section 3 presents the equilibrium analysis of the model. Section 4 investigates the welfare property of the model and extends the basic model. Section 5 compares our result with that of Saijo and Yamato (1999). Section 6 concludes.

\footnotetext{
${ }^{4}$ Myatt and Wallace (2002) and Bitzer and Schröder (2005) also examine the voluntary development of OSS, from a theoretical viewpoint. They explain the incentive for voluntary development of an OSS in terms of the relationship between the benefit and the development cost.
} 


\section{Preliminaries}

Consider an economy in which there are one private good and one public good. Let $N=\{1, \ldots, n\}$ be a set of agents, with $n \geq 2$. Every agent has a preference relation that may be represented by a Leontief utility function. Let $U_{i}\left(Y, x_{i}\right)=\min \left\{Y, x_{i}\right\}$ be a utility function of $i \in N$, in which $Y$ represents the supply of the public good and $x_{i}$ is the private good consumption of $i \in N$. Every agent $i \in N$ has an initial endowment of $w_{i}>0$ of the private good, but none of the public good. The public good is produced from the private good. Suppose that $i \in N$ pays $p_{i}$ units of the private good to produce one unit of the public good: $p_{i}>0$ is the "price" of the public good. The lower $p_{i}$ is, the more efficient agent $i$. Without loss of generality, we assume that $p_{1} \leq \cdots \leq p_{n}$. Let $y_{i} \in \mathbb{R}_{+}$be a supply level of the public good produced by $i \in N$. Then the budget balance condition of $i \in N$ is $x_{i}+p_{i} y_{i}=w_{i} \cdot{ }^{5}$ Let $\left(y_{1} \ldots, y_{n}\right) \in \mathbb{R}_{+}^{n}$ be the profile of contributions to the public good. The quantity of the public good produced in the economy is $Y=\sum_{j \in N} y_{j}$.

We consider a situation in which there exists an opportunity for joint production of the public good, and each agent can decide whether to participate in production. We consider the following two-stage game. In the first stage, agents simultaneously decide whether to participate in providing the public good (participation stage). In the second stage, all agents know the others' participation decisions, and simultaneously decide the quantity of the public good to produce. The nonparticipants can free ride the public good. The formal definition of the game is as follows.

Stage 1. Every agent $i \in N$ chooses whether to participate, simultaneously with the others.

Stage 2. Let $P \subseteq N$ be a set of participants. Each $i \in P$ decides the contribution $y_{i, P} \in$ $\mathbb{R}_{+}$, simultaneously with others. The level of the public good provided by the participants is $Y_{P}=\sum_{j \in P} y_{j, P}$. The payoff to $i \in P$ is $U_{i}\left(Y_{P}, w_{i}-p_{i} y_{i, P}\right)=\min \left\{Y_{P}, w_{i}-p_{i} y_{i, P}\right\}$. The

\footnotetext{
${ }^{5}$ Note that we impose no condition on the order of $\left(w_{i}\right)_{i \in N}$.
} 
payoff to $i \in N \backslash P$ is $U_{i}\left(Y_{P}, w_{i}\right)=\min \left\{Y_{P}, w_{i}\right\}$.

The two-stage public good provision game is known as the (voluntary) participation game, introduced by Saijo and Yamato (1999).

\section{A voluntary participation game: Equilibrium analysis}

\subsection{Nash equilibria in the second-stage game}

We analyze the voluntary participation game by backward induction. We first solve the second-stage game. Let $P \subseteq N$ be a set of participants. Let $y_{i, P}^{*}: \mathbb{R}_{+} \rightarrow \mathbb{R}_{+}$be a best response function of $i \in P$ such that $\sum_{j \in P \backslash\{i\}} y_{j, P} \in \mathbb{R}_{+} \mapsto y_{i, P}^{*}\left(\sum_{j \in P \backslash\{i\}} y_{j, P}\right) \in \mathbb{R}_{+}$. Then

$$
y_{i, P}^{*}\left(\sum_{j \in P \backslash\{i\}} y_{j, P}\right)=\left\{\begin{array}{ll}
\frac{w_{i}-\sum_{j \in P \backslash\{i\}} y_{j, P}}{1+p_{i}} & \text { if } w_{i}>\sum_{j \in P \backslash\{i\}} y_{j, P} \\
0 & \text { otherwise }
\end{array} .\right.
$$

By the definition of the (pure-strategy) Nash equilibrium, $y_{P}^{*} \equiv\left(y_{i, P}^{*}\right)_{i \in P} \in \mathbb{R}_{+}^{\# P}$ is a Nash equilibrium of the second-stage game when $P$ is a set of participants if and only if $y_{i, P}^{*}=y_{i, P}^{*}\left(\sum_{j \in P \backslash\{i\}} y_{j, P}^{*}\right)$ for each $i \in P$. In the voluntary contribution game, a Nash equilibrium is not necessarily an interior solution. Let $C\left(P, y_{P}^{*}\right) \equiv\left\{j \in N \mid y_{j, P}^{*}>0\right\}$ : the set of contributors, participants contributing to the public good, at $y_{P}^{*}$.

Lemma 1 Let $P \subseteq N$ be a set of participants. Let $y_{P}^{*}$ be a Nash equilibrium of the voluntary contribution game. (i) Set $C\left(P, y_{P}^{*}\right)$ is not empty.

(ii) For each $i \in P$

$y_{i, P}^{*}=\left\{\begin{array}{ll}\frac{1}{p_{i}\left(1+\sum_{j \in C\left(P, y_{P}^{*}\right)}\left(1 / p_{j}\right)\right)}\left(w_{i}+w_{i} \sum_{j \in C\left(P, y_{P}^{*}\right)} \frac{1}{p_{j}}-\sum_{j \in C\left(P, y_{P}^{*}\right)} \frac{w_{j}}{p_{j}}\right) & \text { if } i \in C\left(P, y_{P}^{*}\right) \\ 0 & \text { otherwise }\end{array}\right.$.

(iii) $\sum_{j \in P} y_{j, P}^{*}=\frac{\sum_{j \in C\left(P, y_{P}^{*}\right)}\left(w_{j} / p_{j}\right)}{1+\sum_{j \in C\left(P, y_{P}^{*}\right)}\left(1 / p_{j}\right)}$.

(iv) For each $i \in P, y_{i, P}^{*}>0$ if and only if $w_{i}>\sum_{j \in P} y_{j, P}^{*}$. 
Proof. Suppose that $C\left(P, y_{P}^{*}\right)=\emptyset$. For each $i \in P$, if $y_{j, P}^{*}=0$ for each $j \in P \backslash\{i\}$, then $i \in P$ is made better off by providing $w_{i} /\left(1+p_{i}\right)$ units of the public good, which contradicts that $y_{P}^{*}$ is a Nash equilibrium.

Note that $\sum_{j \in P} y_{j, P}^{*}=\sum_{j \in C\left(P, y_{P}^{*}\right)} y_{j, P}^{*}$ and $w_{j} \leq \sum_{j \in C\left(P, y_{P}^{*}\right)} y_{j, P}^{*}$ for each $j \notin C\left(P, y_{P}^{*}\right)$. By (1)

$$
y_{i, P}^{*}=\frac{w_{i}-\sum_{j \in C\left(P, y_{P}^{*}\right) \backslash\{i\}} y_{j, P}}{1+p_{i}} \text { or } y_{i, P}^{*}=\frac{w_{i}-\sum_{j \in C\left(P, y_{P}^{*}\right)} y_{j, P}}{1+p_{i}}+\frac{y_{i, P}^{*}}{1+p_{i}}
$$

for each $i \in C\left(P, y_{P}^{*}\right)$. Thus

$$
y_{i, P}^{*}=\frac{w_{i}}{p_{i}}-\frac{1}{p_{i}} \sum_{j \in C\left(P, y_{P}^{*}\right)} y_{j, P}^{*}
$$

for each $i \in C\left(P, y_{P}^{*}\right)$. Summarizing these conditions over $i \in C\left(P, y_{P}^{*}\right)$ yields

$$
\sum_{j \in C\left(P, y_{P}^{*}\right)} y_{j, P}^{*}=\sum_{j \in C\left(P, y_{P}^{*}\right)} \frac{w_{j}}{p_{j}}-\sum_{j \in C\left(P, y_{P}^{*}\right)} \frac{1}{p_{j}} \sum_{j \in C\left(P, y_{P}^{*}\right)} y_{j, P}^{*} .
$$

Then

$$
\sum_{j \in C\left(P, y_{P}^{*}\right)} y_{j, P}^{*}=\frac{\sum_{j \in C\left(P, y_{P}^{*}\right)}\left(w_{j} / p_{j}\right)}{1+\sum_{j \in C\left(P, y_{P}^{*}\right)}\left(1 / p_{j}\right)}
$$

Substituting (3) into (2), we obtain

$$
y_{i, P}^{*}=\frac{1}{p_{i}\left(1+\sum_{j \in C\left(P, y_{P}^{*}\right)}\left(1 / p_{j}\right)\right)}\left(w_{i}+w_{i} \sum_{j \in C\left(P, y_{P}^{*}\right)} \frac{1}{p_{j}}-\sum_{j \in C\left(P, y_{P}^{*}\right)} \frac{w_{j}}{p_{j}}\right)
$$

for each $i \in C\left(P, y_{P}^{*}\right)$. By (4)

$$
y_{i, P}^{*}=\frac{1}{p_{i}\left(1+\sum_{j \in C\left(P, y_{P}^{*}\right)}\left(1 / p_{j}\right)\right)}\left(w_{i}\left(1+\sum_{j \in C\left(P, y_{P}^{*}\right)} \frac{1}{p_{j}}\right)-\sum_{j \in C\left(P, y_{P}^{*}\right)} \frac{w_{j}}{p_{j}}\right),
$$

which shows (iv).

Lemma 2 Let $P \subseteq N$ be a set of participants. Suppose that $w=w_{i}$ for each $i \in P$. Then

$$
\sum_{j \in C\left(P, y_{P}^{*}\right)} y_{j, N}^{*}=\frac{\sum_{j \in C\left(P, y_{P}^{*}\right)}\left(1 / p_{j}\right)}{1+\sum_{j \in C\left(P, y_{P}^{*}\right)}\left(1 / p_{j}\right)} w<w,
$$

which implies that $C\left(P, y_{P}^{*}\right)=P$. 
Proof. It is immediately seen from (3) that (5) holds. By (5) and (iv) in Lemma 1, $C\left(P, y_{P}^{*}\right)=P$.

A Nash equilibrium in the voluntary contribution game when $P \subseteq N$ is a set of participants is not necessarily an interior solution; every agent in $P$ contributes a positive amount of a public good at a Nash equilibrium. Whereas the Nash equilibrium for this game is interior if every participant has the same initial endowment of the private good, as Lemma 2 shows, it is not necessarily interior otherwise. We must note a possible case in which an efficient agent makes a smaller contribution than an inefficient agent, depending on the distribution of the initial endowments of the private good, as Example 1 shows.

Example 1 Let $n=2$, with $w_{1}<w_{2}$ and $p_{1}<p_{2}$. If $\left(1+p_{2}\right) w_{1}-w_{2}>0$

$$
y_{1}^{*}=\frac{\left(1+p_{2}\right) w_{1}-w_{2}}{p_{1}+p_{2}+p_{1} p_{2}} \text { and } y_{2}^{*}=\frac{\left(1+p_{1}\right) w_{2}-w_{1}}{p_{1}+p_{2}+p_{1} p_{2}},
$$

otherwise

$$
y_{1}^{*}=0 \text { and } y_{2}^{*}=\frac{w_{2}}{1+p_{2}} .
$$

When a Nash equilibrium is interior, by (6), we have

$$
y_{1}^{*}-y_{2}^{*}=\frac{\left(2+p_{2}\right) w_{1}-\left(2+p_{1}\right) w_{2}}{p_{1}+p_{2}+p_{1} p_{2}} .
$$

This is negative if and only if $w_{2}>\left(2+p_{2}\right) w_{1} /\left(2+p_{1}\right)\left(>w_{1}\right)$. There is a possibility that the efficient provider, agent 1, provides fewer public goods than the other agents, regardless of whether the Nash equilibrium is interior.

From (4), we find that whether an agent becomes a contributor depends on the amount of the initial endowment of the private good. The first fraction in (4) is always positive because $p_{i}>0$ for any $i$. The sign of $y_{i, P}^{*}$ is, therefore, the same as that of the value between the parentheses, which is increasing in $w_{i}$. This means that if agent $i$ with $w_{i}$ provides a positive amount of the public good, so does agent $k$ with $w_{k}\left(>w_{i}\right)$. 
Proposition 1 For each $P \subseteq N$ and each pair $i, k \in P$, if $y_{i, P}^{*}>0$ and $w_{k} \geq w_{i}$, then $y_{k, P}^{*}>0$.

Proof. If the value between the parentheses of $y_{k, P}^{*}$ is larger than that of $y_{i, P}^{*}, y_{k, P}^{*}>0$.

The difference between the values is given as

$$
\begin{aligned}
& \left(w_{k}+w_{k} \sum_{j \in C\left(P, y_{P}^{*}\right)} \frac{1}{p_{j}}-\sum_{j \in C\left(P, y_{P}^{*}\right)} \frac{w_{j}}{p_{j}}\right)-\left(w_{i}+w_{i} \sum_{j \in C\left(P, y_{P}^{*}\right)} \frac{1}{p_{j}}-\sum_{j \in C\left(P, y_{P}^{*}\right)} \frac{w_{j}}{p_{j}}\right) \\
& =\left(w_{k}-w_{i}\right)\left(1+w_{k} \sum_{j \in C\left(P, y_{P}^{*}\right)} \frac{1}{p_{j}}\right) .
\end{aligned}
$$

The last equation is nonnegative when $w_{k} \geq w_{i}$. The proposition holds.

The value between the parentheses in (4) reflects the decision of agent $i$ on whether to contribute. The first fraction in (4) reflects the significance of the contribution from agent $i$. As the value of $p_{i}$ decreases, the first fraction in (4) increases.

\subsection{Who participates? Equilibrium analysis of the first stage}

We examine the first-stage game induced by $y_{P}^{*}$ in Lemma 1 for each $P \subseteq N$.

We first provide basic properties of the set of contributors and the level of the public good in Lemmas 3, 4, and 5. Lemma 3 shows that the level of the public good is the same in the case in which the entire $P \subseteq N$ is a set of participants and the case in which its set of contributors $C\left(P, y_{P}^{*}\right)$ is itself a set of participants: the noncontributors in $P$ never affect the decision of the contributors.

Lemma 3 For each $P \subseteq N, \sum_{j \in P} y_{j, P}^{*}=\sum_{j \in C\left(P, y_{P}^{*}\right)} y_{j, C\left(P, y_{P}^{*}\right)}^{*}$.

Proof. By Lemma 1, for each $i \in C\left(P, y_{P}^{*}\right), y_{i, P}^{*}$ depends on $\sum_{j \in C\left(P, y_{P}^{*}\right)}\left(1 / p_{j}\right)$ and $\sum_{j \in C\left(P, y_{P}^{*}\right)}\left(w_{j} / p_{j}\right)$. These values do not change if $C\left(P, y_{P}^{*}\right)$ is a set of participants. Thus, $y_{i, P}^{*}=y_{i, C\left(P, y_{P}^{*}\right)}^{*}$ for each $i \in C\left(P, y_{P}^{*}\right)$. 
Lemmas 4 and 5 show how the set of contributors and the level of the public good change if the set of participants is expanded. Lemma 4 examines the effect of the expansion by agent $i$ such that $w_{i}>\sum_{j \in P} y_{j, P}^{*}$ (influential agent), while Lemma 5 examines the effects by agent $i$ such that $w_{i} \leq \sum_{j \in P} y_{j, P}^{*}$ (noninfluential agent).

Lemma 4 Let $P \subsetneq N$ and let $i \in N \backslash P$. Suppose that $w_{i}>\sum_{j \in P} y_{j, P}^{*}$. Then

(4.1) $i \in C\left(P \cup\{i\}, y_{P \cup\{i\}}^{*}\right)$,

(4.2) $\sum_{j \in P \cup\{i\}} y_{j, P \cup\{i\}}^{*}>\sum_{j \in P} y_{j, P}^{*}$, and

(4.3) $C\left(P \cup\{i\}, y_{P \cup\{i\}}^{*}\right)=C\left(P \cup\{i\}, y_{P \cup\{i\}}^{*}\right) \cap C\left(P, y_{P}^{*}\right) \cup\{i\}$.

The proof is in the appendix. Agent $i$ such that $w_{i}>\sum_{j \in P} y_{j, P}^{*}$ is influential on $P$ in the sense that $i$ becomes a contributor and his/her participation increases the level of the public good as (4.1) and (4.2) show. By (4.3), the noncontributors before the participation of such an agent never become contributors after this participation: no agents in $P \backslash C\left(P, y_{P}^{*}\right)$ change their behavior as a result of this participation.

Lemma 5 For each $P \subsetneq N$ and each $i \in N \backslash P$, if $w_{i} \leq \sum_{j \in P} y_{j, P}^{*}$, then $i \notin C(P \cup$ $\left.\{i\}, y_{P \cup\{i\}}^{*}\right)$ and $C\left(P \cup\{i\}, y_{P \cup\{i\}}^{*}\right)=C\left(P, y_{P}^{*}\right)$.

The proof is in the appendix. By Lemma 5, no agent $i$ such that $w_{i} \leq \sum_{j \in P} y_{j, P}^{*}$ changes the set of contributors and the level of the public good even if he/she participates in $P$.

Proposition 2 presents a condition under which each agent participates in public good provision.

Proposition 2 For each $P \subsetneq N$ and each $i \in N \backslash P$, if $w_{i}>\sum_{j \in P} y_{j, P}^{*}$, then $i$ is made better off by joining $P$. Otherwise, $i$ is indifferent between participation and nonparticipation. 
Proof. By (4.1) and (4.2) of Lemma 4, $w_{i}>\sum_{j \in P \cup\{i\}} y_{j, P \cup\{i\}}^{*}>\sum_{j \in P} y_{j, P}^{*}$ for each $i \in N \backslash P$ such that $w_{i}>\sum_{j \in P} y_{j, P}^{*}$. If such an agent $i$ chooses to participate, his/her payoff is $\sum_{j \in P \cup\{i\}} y_{j, P \cup\{i\}}^{*}$ and if he/she chooses not to participate, his/her payoff is $\sum_{j \in P} y_{j, P}^{*}$.

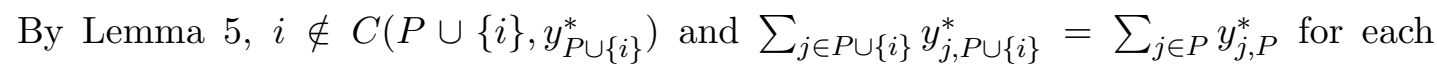
$i \in N \backslash P$ such that $w_{i} \leq \sum_{j \in P} y_{j, P}^{*}$. Thus, his/her payoff is $w_{i}$, regardless of whether he/she participates.

Let $P \subsetneq N$. Let $i \notin P$ be such that $w_{i}>\sum_{j \in P} y_{j, P}^{*}$ and let $k \notin P$ be such that $w_{k} \leq \sum_{j \in P} y_{j, P}^{*}$. By Proposition 2 and (4.1) of Lemma 4, whereas $i$ is willing to participate and make a contribution, $k$ is indifferent between participating and not participating. Because $i$ has a Leontief preference, he/she may be better off by increasing the level of the public good. If $i$ participates in $P$, he/she can contribute an adequate amount of the private good in a way that increases the public good provision ((4.2) of Lemma 4). Although $k$ has the same preference, he/she cannot increase his/her payoff any further. If $k$ participates and contributes a positive amount, $k$ is made worse off. Even if $k$ participates, he/she makes no contribution; thus, participating and not participating are equivalent for $k$.

By the definition of a Nash equilibrium, $P \subseteq N$ is a set of participants supported at a Nash equilibrium if and only if (i) $U_{i}\left(\sum_{j \in P} y_{j, P}^{*}, w_{i}-p_{i} y_{j, P}^{*}\right) \geq U_{i}\left(\sum_{j \in P \backslash\{i\}} y_{i, P \backslash\{i\}}^{*}, w_{i}-\right.$ $\left.p_{i} y_{i, P \backslash\{i\}}^{*}\right)$ for each $i \in P$ and (ii) $U_{i}\left(\sum_{j \in P} y_{j, P}^{*}, w_{i}\right) \geq U_{i}\left(\sum_{j \in P \cup\{i\}} y_{i, P \cup\{i\}}^{*}, w_{i}-p_{i} y_{i, P \cup\{i\}}^{*}\right)$ for each $i \in N \backslash P$. Condition (i) means that no participant can be made better off by deviating to nonparticipation and (ii) means that no nonparticipant can be made better off by joining $P$. When agents have a Leontief preference, these conditions are equivalent to (i') and (ii') in Proposition 3.

Proposition 3 For each $P \subseteq N, P$ is a Nash equilibrium set of participants if and only if (i') $w_{i}>\sum_{j \in P \backslash\{i\}} y_{j, P \backslash\{i\}}^{*}$ for each $i \in C\left(P, y_{P}^{*}\right)$ and (ii') $w_{i} \leq \sum_{j \in P} y_{j, P}^{*}$ for each $i \in N \backslash P$. 
Proof. (sufficiency) By Proposition 2, no $i \in C\left(P, y_{P}^{*}\right)$ and $j \in N \backslash P$ have an incentive to change participation decisions. For each $k \in P \backslash C\left(P, y_{P}^{*}\right), y_{k, P}^{*}=0$; hence, $w_{k} \leq$ $\sum_{j \in P} y_{j, P}^{*}$. Because $C\left(P, y_{P}^{*}\right)=C\left(P \backslash\{k\}, y_{P \backslash\{k\}}^{*}\right)$, then $\sum_{j \in P} y_{j, P}^{*}=\sum_{j \in P \backslash\{k\}} y_{j, P \backslash\{k\}}^{*}$. Thus, $k$ obtains a payoff $w_{k}$ irrespective of his/her participation decision.

(necessity) Suppose that there is $i \in C\left(P, y_{P}^{*}\right)$ such that $w_{i} \leq \sum_{j \in P \backslash\{i\}} y_{j, P \backslash\{i\}}^{*}$. Because $i \in C\left(P, y_{P}^{*}\right)$ implies that $\sum_{j \in P} y_{j, P}^{*}<w_{i}$, then $\sum_{j \in P} y_{j, P}^{*}<w_{i} \leq \sum_{j \in P \backslash\{i\}} y_{j, P \backslash\{i\}}^{*}$. Thus, $i$ 's payoff if he/she participates, $\sum_{j \in P} y_{j, P}^{*}$, is less than that if he/she does not participate, $w_{i}$. In conclusion, $i$ is better off switching from participation to nonparticipation, a contradiction. By Proposition 2, if there is $i \in N \backslash P$ such that $w_{i}>\sum_{j \in P} y_{j, P}^{*}$, then $i$ is better off participating, which is a contradiction. Thus, (i') and (ii') hold.

By Proposition 3, to check whether $P$ is a Nash equilibrium set of participants, it is sufficient to check whether $P \subseteq N$ satisfies (i') and (ii') in the proposition. By Propositions 2 and 3 , if $P \subseteq N$ is a Nash equilibrium set of participants, then every agent outside $P$ is indifferent between participating and not participating. Because of this indifference, a Nash equilibrium set of participants may not be unique. Proposition 4 identifies all sets of participants that are supported at a Nash equilibrium.

Proposition 4 For each $P \subseteq N, P$ is a Nash equilibrium set of participants if and only if $C\left(N, y_{N}^{*}\right) \subseteq P \subseteq N$.

The proof is in the appendix. In the voluntary participation game, there are generally $\#\left\{P \mid C\left(N, y_{N}^{*}\right) \subseteq P \subseteq N\right\}$ subgame-perfect Nash equilibria because the second-stage game has the unique Nash equilibrium for each (nonempty) set of participants. However, each agent in $N \backslash C\left(N, y_{N}^{*}\right)$ is indifferent between participating and not participating and he/she contributes nothing at the equilibria even if he/she participates. Thus, the allocation supported at subgame-perfect Nash equilibria is unique. 


\section{Welfare analysis}

We discuss the welfare properties of the allocations attained at the subgame-perfect Nash equilibria of the voluntary participation game. Some of the initial endowments are transferable among agents and others are not. For example, money, which is a resource for development of OSS, is an example of transferable resources. We investigate whether the equilibrium allocation is Pareto efficient in both cases.

We first consider the case of nontransferable endowments. Definition 1 provides concepts of the feasible allocation and the Pareto efficiency in the economy in which agents cannot transfer the private good to each other.

Definition 1 (i) An allocation $\left(Y,\left(y_{i}, x_{i}\right)_{i \in N}\right)$ is individually feasible if $w_{i} \geq p_{i} y_{i}+x_{i}$ for each $i \in N$ and $Y=\sum_{i \in N} y_{i}$. (ii) An allocation $\left(Y,\left(y_{i}, x_{i}\right)_{i \in N}\right)$ is Pareto efficient within the set of individually feasible allocations - henceforth, $i$-Pareto efficient for short-if there is no individually feasible allocation $\left(Y^{\prime},\left(y_{i}^{\prime}, x_{i}^{\prime}\right)_{i \in N}\right)$ such that $U_{i}\left(Y^{\prime}, x_{i}^{\prime}\right) \geq U_{i}\left(Y, x_{i}\right)$ for all $i \in N$ with strict inequality for at least one $i \in N$.

Proposition 5 The allocation at the subgame-perfect Nash equilibria of the voluntary participation game is i-Pareto efficient.

Proof. At the subgame-perfect Nash equilibria, the payoff of agent $i$ is $\sum_{j \in C\left(N, y_{N}^{*}\right)} y_{j, N}^{*}$ if $i \in C\left(N, y_{N}^{*}\right)$ and $w_{i}$ if $i \in N \backslash C\left(N, y_{N}^{*}\right)$. First, no $i \in N \backslash C\left(N, y_{N}^{*}\right)$ is made better off by the switch of allocations. By (iv) of Lemma $1, w_{i} \leq \sum_{j \in C\left(N, y_{N}^{*}\right)} y_{j, N}^{*}$. To increase the payoff to $i \in N \backslash C\left(N, y_{N}^{*}\right)$, it is necessary that $i$ 's consumption of the private good increases beyond $w_{i}$. However, the consumption of the private good is at most $w_{i}$ within the set of individually feasible allocations. Second, we show that if $k \in C\left(N, y_{N}^{*}\right)$ is made better off, then the other agent is made worse off. For $k \in C\left(N, y_{N}^{*}\right), \sum_{j \in C\left(N, y_{N}^{*}\right)} y_{j, N}^{*}=w_{k}-p_{k} y_{k, N}^{*}$, where the right-hand side is $k$ 's consumption of the private good. Because $k$ has a Leontief utility function, to improve his/her payoff, it is necessary to reduce his/her contribution of 
the public good, which induces an increase in the private good consumption of $k$, and other agents increase their contribution of the public good more than the reduction. However, the change of contribution of the others decreases their payoffs. In summary, no Pareto improvement is possible.

The welfare property of the subgame-perfect Nash equilibria is related to the result of Kukushkin (1992). Using an abstract one-shot game, he characterizes a condition for utility functions under which there is a Nash equilibrium with Pareto efficiency. The Leontief utility functions satisfy his condition. However, he does not clarify the equilibrium behavior (contribution and participation) of agents because his model is abstract and does not examine a concrete model of a public good economy. Unlike the findings of Kukushkin (1992), our result shows the behavior of each agent. For example, Proposition 1 shows that the participation decision of the agent depends solely on his/her initial endowment of the private good; Proposition 5, together with Proposition 4, shows that the partial participation of agents, not necessarily that of all agents, achieves the i-Paretoefficient allocation at the equilibrium of the voluntary participation game. In Saijo and Yamato's $(1999,2010)$ model, the i-Pareto-efficient allocation is achieved only if all agents participate.

We next consider the case of transferable endowments. Definition 2 describes feasibility and Pareto efficiency when agents can transfer their endowments to others.

Definition 2 (i) An allocation $\left(Y,\left(y_{i}, x_{i}\right)_{i \in N}\right)$ is socially feasible if $\sum_{i \in N} w_{i} \geq \sum_{i \in N}\left(p_{i} y_{i}+x_{i}\right)$ and $Y=\sum_{i \in N} y_{i}$. (ii) An allocation $\left(Y,\left(y_{i}, x_{i}\right)_{i \in N}\right)$ is Pareto efficient within the set of socially feasible allocations - henceforth, s-Pareto efficient for short-if there is no socially feasible allocation $\left(Y^{\prime},\left(y_{i}^{\prime}, x_{i}^{\prime}\right)_{i \in N}\right)$ such that $U_{i}\left(Y^{\prime}, x_{i}^{\prime}\right) \geq U_{i}\left(Y, x_{i}\right)$ for all $i \in N$ with strict inequality for at least one $i \in N$.

We exemplify that the allocation at the subgame-perfect Nash equilibrium in the voluntary participation game is not necessarily s-Pareto efficient. 
Example 1' Let $n=2$, with $w_{1}<w_{2}$ and $p_{1}<p_{2}$. Without transfers, by (6)

$$
y_{1}^{*}=\frac{\left(1+p_{2}\right) w_{1}-w_{2}}{p_{1}+p_{2}+p_{1} p_{2}}, y_{2}^{*}=\frac{\left(1+p_{1}\right) w_{2}-w_{1}}{p_{1}+p_{2}+p_{1} p_{2}}, \text { and } \sum_{i \in N} y_{i}^{*}=\frac{p_{1} w_{2}+p_{2} w_{1}}{p_{1}+p_{2}+p_{1} p_{2}},
$$

if $\left(1+p_{2}\right) w_{1}-w_{2} \geq 0$ and $\left(1+p_{1}\right) w_{2}-w_{1} \geq 0$. We assume that the two equalities hold. When agent 2 transfers his/her endowment of the private good by $t \in\left[0, w_{2}\right]$ to agent 1 , their ex post endowments of the private good are $w_{1}^{\prime}=w_{1}+t$ and $w_{2}^{\prime}=w_{2}-t$. The $e x$ post level of the public good is

$$
\frac{p_{1} w_{2}+p_{2} w_{1}+\left(p_{2}-p_{1}\right) t}{p_{1}+p_{2}+p_{1} p_{2}}
$$

if $\left(1+p_{2}\right) w_{1}^{\prime}-w_{2}^{\prime} \geq 0$ and $\left(1+p_{1}\right) w_{2}^{\prime}-w_{1}^{\prime} \geq 0$. For $t \in\left[0, w_{2}\right]$, only the latter inequality can be binding. This is rewritten as

$$
\left(1+p_{1}\right) w_{2}^{\prime}-w_{1}^{\prime}=\left(1+p_{1}\right) w_{2}-w_{1}-\left(2+p_{1}\right) t \geq 0 \text { or } t \leq \frac{\left(1+p_{1}\right) w_{2}-w_{1}}{2+p_{1}} .
$$

For $t$ satisfying the inequality, the ex post level of the public good is increasing in $t$. The maximum level of the public good is $\left(w_{1}+w_{2}\right) /\left(2+p_{1}\right)$, which is equivalent to the utility levels of the two agents. Because $\sum_{j \in N} y_{j}^{*}<\left(w_{1}+w_{2}\right) /\left(2+p_{1}\right)$, the allocation attained by the transfer Pareto-dominates the allocation with (7).

The s-Pareto efficiency of the ex post allocation through the transfer can be easily checked by Proposition 6 below.

Proposition 6 provides a necessary and sufficient condition under which the voluntary participation game has a subgame-perfect Nash equilibrium that achieves s-Pareto efficiency.

Proposition 6 Suppose that $p_{1}<p_{j}$ for each $j \in N \backslash\{1\}$. Let $\left(Y^{*},\left(y_{i}^{*}, x_{i}^{*}\right)_{i \in N}\right)$ be a socially feasible allocation supported at a subgame-perfect Nash equilibrium of the voluntary participation game. Then, $\left(Y^{*},\left(y_{i}^{*}, x_{i}^{*}\right)_{i \in N}\right)$ is s-Pareto efficient if and only if agent 1 is the only contributor. 
Proof. (sufficiency) At the equilibrium, $x_{1}^{*}=w_{1}-p_{1} y_{1}^{*}=y_{1}^{*}$ and $x_{j}^{*}=w_{j} \leq y_{1}^{*}$ for each $j \in N \backslash\{1\}$. Under these conditions, to increase the payoff of agent 1 , it is necessary to increase both agent 1's consumption of the private good and the level of the public good using the others' initial endowments of the private good. However, these other agents are made worse off because their consumption of the private good decreases.

(necessity) Suppose, to the contrary, that agent 1 is not the only contributor, which implies that there is $j \in N \backslash\{1\}$ that is a contributor, $y_{j}^{*}>0$. Consider the following allocation $\left(Y^{\prime},\left(y_{i}^{\prime}, x_{i}^{\prime}\right)_{i \in N}\right)$ such that $Y^{\prime}=\sum_{i \in N} y_{i}^{\prime}$ and for all $k \in N$

$$
x_{k}^{\prime}=\left\{\begin{array}{ll}
x_{1}^{*}+\frac{p_{j}-p_{1}}{2} y_{j}^{*} & \text { if } k=1 \\
x_{k}^{*} & \text { otherwise }
\end{array} \text { and } y_{k}^{\prime}=\left\{\begin{array}{ll}
y_{1}^{*}+y_{j}^{*}+\frac{p_{j}-p_{1}}{2 p_{1}} y_{j}^{*} & \text { if } k=1 \\
0 & \text { if } k=j \\
y_{k}^{*} & \text { otherwise }
\end{array} .\right.\right.
$$

Because $\sum_{i \in\{1, j\}}\left(x_{i}^{\prime}+p_{i} y_{i}^{\prime}\right)=\sum_{i \in\{1, j\}}\left(x_{i}^{*}+p_{i} y_{i}^{*}\right)$, this allocation is socially feasible. Because $Y^{\prime}>Y^{*}$ and $x_{1}^{\prime}>x_{1}^{*}$, agent 1 is made better off by switching from $\left(Y^{*},\left(y_{i}^{*}, x_{i}^{*}\right)_{i \in N}\right)$ to $\left(Y^{\prime},\left(y_{i}^{\prime}, x_{i}^{\prime}\right)_{i \in N}\right)$. Note that by this switch, the amount of the public good increases and the consumption of the private good of agent $k$ is the same for each $k \in N \backslash\{1\}$. Thus, no agent $k \in N \backslash\{1\}$ is made worse off by the switch. This is a contradiction.

In the voluntary participation game, the endowment transfer between agents is not allowed. Thus, even if an agent has an efficient technology (a low price), if he/she does not have enough endowments of the private good, then he/she may not be a contributor. There is a possibility that an efficient technology is not used to provide a public good because of the lack of initial endowments. This possibility becomes a source of allocative inefficiency.

By Proposition 6, the following question naturally comes to mind: is the s-Paretoefficient allocation achieved as an equilibrium of a game in which agents can freely transfer their endowments to each other? We extend a voluntary participation game by allowing the transfer of endowments among agents according to the endogenous-game approach 
of Jackson and Wilkie (2005) and examine cases in which s-Pareto-efficient allocation is achieved through the transfer. A voluntary participation game with side payments is a game in which there is a side payment stage among agents prior to the voluntary participation game. The formal statement of the game is as follows.

Definition 3 (A voluntary participation game with side payments) Let $w_{i} \geq 0$ be an initial endowment of the private good of agent $i \in N$. In the voluntary participation game with side payments, each $i \in N$ first simultaneously chooses $t_{i} \equiv\left(t_{i j}\right)_{j \in N} \in \mathbb{R}_{+}^{n}$ such that $t_{i i}=0$ and $\sum_{j \in N} t_{i j} \leq w_{i}$, where $t_{i j} \geq 0$ represents the amount of the private good transferred from $i \in N$ to $j \in N$. At the end of this stage, each $i \in N$ has $w_{i}^{\prime} \equiv w_{i}+\sum_{j \in N \backslash\{i\}}\left(t_{j i}-t_{i j}\right)$. The second and third stages are equivalent to the voluntary participation game when each $i \in N$ has $w_{i}^{\prime}$ units of the private good at the start of the participation decision.

We establish a sufficient condition under which the voluntary transfer of the private good among agents achieves the s-Pareto-efficient allocation in a subgame-perfect Nash equilibrium of the voluntary participation game with side payments.

Proposition 7 An s-Pareto-efficient allocation can be achieved at a subgame- perfect Nash equilibrium of the voluntary participation game with side payments if

$$
\left(n+p_{1}\right) w_{i}>\sum_{j \in N} w_{j}>\frac{n+p_{1}}{1+p_{2}} w_{i} \text { for each } i \in N \backslash\{1\} .
$$

Proof. Let $\left(Y^{e},\left(y_{j}^{e}, x_{j}^{e}\right)_{j \in N}\right)$ be a feasible allocation such that

$$
\begin{aligned}
y_{1}^{e} & =\frac{\sum_{j \in N} w_{j}}{n+p_{1}}, y_{j}^{e}=0 \text { for each } j \in N \backslash\{1\}, \quad Y^{e}=y_{1}^{e}, \\
\text { and } \quad x_{j}^{e} & =Y^{e} \text { for all } j \in N .
\end{aligned}
$$

Let $\left(t_{j i}^{e}\right)_{j, i \in N}$ be a transfer scheme such that

$$
t_{j i}^{e}=\left\{\begin{array}{ll}
w_{j}-x_{j}^{e}=w_{j}-y_{1}^{e} & \text { if } j \neq 1 \text { and } i=1 \\
0 & \text { if } j \neq 1 \text { and } i \neq 1 \text { or } j=1
\end{array} .\right.
$$


Given $\left(t_{j i}^{e}\right)_{j, i \in N}$, each $i \in N$ has $w_{i}^{e}=\left(1+p_{1}\right) y_{1}^{e}$ of the private good if $i=1$ and $w_{i}^{e}=y_{1}^{e}$ otherwise at the beginning of the second-stage game and $\left(Y^{e},\left(y_{j}^{e}, x_{j}^{e}\right)_{j \in N}\right)$ is attained at the equilibrium outcome of the second and third stages. By Proposition 6 , because agent 1 is the only contributor at $\left(Y^{e},\left(y_{j}^{e}, x_{j}^{e}\right)_{j \in N}\right)$, it is s-Pareto efficient.

We show that the transfer scheme is feasible, which means that $t_{j 1}^{e}>0$ for each $j \in N \backslash\{1\}$, and it is supported at a subgame-perfect Nash equilibrium in the voluntary participation game with side payments. Clearly, for each $i \in N \backslash\{1\}, t_{j 1}^{e}>0$ if and only if

$$
w_{i}-\frac{\sum_{j \in N} w_{j}}{n+p_{1}}=\frac{1}{n+p_{1}}\left(\left(n+p_{1}\right) w_{i}-\sum_{j \in N} w_{j}\right)>0 .
$$

We prove that no agent has an incentive to reduce their amount of transfer given the transfers of the other agents. Now suppose that some agent $j \in N \backslash\{1\}$ reduces the level of transfer $t_{j 1}^{e}$ by $z_{j}$ and allocates $z_{j}$ to the other $n-1$ agents including agent $j$. Let $\left(t_{j i}^{\prime}\right)_{i \in N}$ be such a transfer scheme of $j: t_{j 1}^{\prime}=t_{j 1}^{e}-z_{j}, \sum_{i \in N \backslash\{1\}} t_{j i}^{\prime}=z_{j}$, and $t_{j i}^{\prime} \geq 0 .{ }^{6}$ Because $j$ has a Leontief utility function, $j$ 's payoff when $j$ deviates is at most the (aggregate) level of the public good. We first identify the ceiling of $j$ 's payoff by deviating, and second prove that the ceiling of $j$ 's payoff is no greater than $y_{1}^{e}$, which is $j$ 's payoff before deviation.

The deviation by $j$ may change the set of contributors $C\left(N, y_{N}^{*}\right)$. Let $Q$ be the changed contributor set. By Lemma 1, for this changed set, the sum of the public good $\sum_{j \in Q} y_{j, Q}^{*}$ is given as

$$
\sum_{i \in Q} y_{i, Q}^{*}=\frac{\sum_{i \in Q}\left(w_{i}^{\prime} / p_{i}\right)}{1+\sum_{i \in Q}\left(1 / p_{i}\right)},
$$

where $w_{1}^{\prime}=w_{1}^{e}-z_{j}$ and $w_{i}^{\prime}=w_{i}^{e}+t_{j i}^{\prime}$ for each $i \in N \backslash\{1\}$. Note that if agent $i \in N \backslash\{1, j\}$ with $t_{j i}^{\prime}>0$ does not contribute, agent $j$ can increase the level of the public good by ceasing to transfer $t_{j i}^{\prime}$ to $i$ and using it for provision of the public good. Thus, there is room to increase the amount of the public good (or the ceiling of $j$ 's payoff by deviating). Therefore, every $i \in N \backslash\{1, j\}$ such that $t_{j i}^{\prime}>0$ must be a contributor: $i \in Q$.

\footnotetext{
${ }^{6}$ By the formal definition of the transfer, $t_{j j}^{\prime}=0$. However, by this proof, we interpret that $t_{j j}^{\prime}=$ $z_{j}-\sum_{i \in N \backslash\{1, j\}} t_{j i}$ for notational brevity. Of course, $t_{j j}^{\prime} \geq 0$ in this notation.
} 
First, suppose that agent 1 contributes. The sum of the public good is

$$
\begin{aligned}
\sum_{k \in Q} y_{k, Q}^{*}= & \frac{\frac{\left(1+p_{1}\right) y_{1}^{e}-\sum_{k \in Q \backslash\{1\}} t_{j k}^{\prime}}{p_{1}}+\sum_{k \in Q \backslash\{1\}} \frac{y_{1}^{e}+t_{j k}^{\prime}}{p_{k}}}{1+\sum_{k \in Q}\left(1 / p_{k}\right)} \\
= & y_{1}^{e}+\frac{\sum_{k \in Q \backslash\{1\}}\left(\frac{1}{p_{k}}-\frac{1}{p_{1}}\right) t_{j k}^{\prime}}{1+\sum_{k \in Q}\left(1 / p_{k}\right)} \leq y_{1}^{e}
\end{aligned}
$$

because $1 / p_{k}-1 / p_{1}=\left(p_{1}-p_{k}\right) /\left(p_{1} p_{k}\right) \leq 0$. Thus, $j$ cannot be made better off by changing to $t_{j}^{\prime}$.

Second, suppose that agent 1 does not contribute. The level of the public good is

$$
\sum_{k \in Q} y_{k, Q}^{*}=\frac{\sum_{k \in Q} \frac{y_{1}^{e}+t_{j k}^{\prime}}{p_{k}}}{1+\sum_{k \in Q}\left(1 / p_{k}\right)} .
$$

Suppose that agent $l$ has the lowest price among members of $Q: p_{l}=\min _{i \in Q} p_{i}$. Allocating some of $t_{j k}^{\prime}$ such that $k \in N \backslash\{1, j, l\}$ to agent $l$ increases the level of the public good. By gradually reducing the value of $t_{j k}^{\prime}$ and allocating it to agent $l$, either agent $k$ does not contribute or the value of $t_{j k}^{\prime}$ becomes zero. In the former situation, for the same reason above, agent $j$ receives the rest of it and expends it to make the public and private goods. Therefore, $t_{j k}^{\prime}=0$ for each $k \in N \backslash\{1, j, l\}$. After the procedure, agent $j$ allocates all of $z_{j}$ to agent $l$. In this situation, there are two possibilities: (1) only agent $l$ contributes, or (2) all agents except 1 contribute.

Case (1). Given the reduction, if only agent $l$ contributes, the level of the public good is

$$
y_{l,\{l\}}=\frac{\left(y_{1}^{e}+z_{j}\right) / p_{l}}{1+1 / p_{l}}=\frac{y_{1}^{e}+z_{j}}{1+p_{l}} .
$$

This is smaller than $y_{1}^{e}$ if and only if $z_{j}<p_{l} y_{1}^{e}$. Note that $z_{j}$ must satisfy $z_{j} \leq w_{j}-y_{1}^{e}$ because $t_{j 1}=w_{j}-y_{1}^{e}$. If $p_{l} y_{1}^{e}$ is larger than $w_{j}-y_{1}^{e}, y_{l}$ is smaller than $y_{1}^{e}$. The condition is

$$
p_{l} y_{1}^{e}-\left(w_{j}-y_{1}^{e}\right)>0 \quad \text { or } \quad y_{1}^{e}>\frac{w_{j}}{1+p_{l}}
$$


Case (2). Given the reduction, if all agents except agent 1 contribute, the total amount of the public good is:

$$
\sum_{k \in N} y_{k, Q}=\frac{y_{1}^{e} \sum_{k \in N \backslash\{1\}} 1 / p_{k}+z_{j} / p_{l}}{1+\sum_{k \in N \backslash\{1\}} 1 / p_{k}} .
$$

This is smaller than $y_{1}^{e}$ if and only if $z_{j}<p_{l} y_{1}^{e}$. This inequality is the same as that in the first case. $\sum_{k \in N} y_{k, N}$ is smaller than $y_{1}^{e}$ if and only if $y_{1}^{e}>w_{j} /\left(1+p_{l}\right)$.

From the discussion, we find that no agent $j \in N \backslash\{1\}$ has an incentive to reduce its transfer if and only if

$$
y_{1}^{e}>\frac{w_{j}}{1+p_{l}} \text { or } \sum_{j \in N} w_{j}>\frac{n+p_{1}}{1+p_{l}} w_{j},
$$

which is satisfied by (9) because $p_{2}$ is the second- lowest value among $\left(p_{i}\right)_{i \in N}$. Trivially, if $j \in N \backslash\{1\}$ increases transfer to agent 1 from $t_{j 1}^{e}$, then $j$ 's payoff decreases.

Condition (9) shows that if the sum of the initial endowments of the private good is neither "too large" nor "too small," then the voluntary transfer of the private good can achieve the s-Pareto-efficient allocation. As we can see in (10), the upper bound of the sum of the initial endowments of the private good guarantees that endowment transfer in the proof is feasible. Without this condition, there is no agent except 1 that can transfer sufficient of the private good, so that agent 1 is the only contributor and the s-Paretoefficient allocation is achieved. The lower bound of the sum of the initial endowments of the private good prevents agents except agent 1 from deviating from the transfer scheme transfer constructed in the proof. In particular, it plays an important role where an agent transfers a large amount of the private good to agent 1 . When such an agent ceases the transfer to agent 1 and reallocates the goods, the set of contributors may change drastically, which may in turn affect the supply of the public good. This case corresponds to Cases (1) and (2) in the proof. In these cases, agent 1 ceases to contribute because agent $j$ deviates and ceases transferring the private good. Without this condition, the supply of the public good may be increased following deviation, which implies that the deviant is made better off by reducing the transfer to agent 1 . 
Although condition (9) may appear to be restrictive, we must note the following aspects. First, some restriction for the initial distribution of the private good is needed for the Pareto- improving transfer scheme to be supported at a subgame-perfect Nash equilibrium. If there is coercion to execute the transfer scheme, then it would be easier to obtain the s-Pareto-efficient allocation through the transfer. In fact, as in the proof of Proposition 6 , it is possible to Pareto improve the outcome supported at the subgame-perfect Nash equilibrium by transfer schemes. However, when there is no outside authority to compel the transfer scheme and agents can freely transfer the initial endowments of the private good, it is a problem whether agents will voluntarily execute the Pareto-improving transfer scheme. The following example shows that if condition (9) is violated, the Paretoimproving transfer scheme cannot be executed without coercion.

Example 2 Consider the case in which $n=2, p_{1}<p_{2}$, and $\left(1+p_{2}\right) w_{1}<w_{2}$. In this case, without transfers, $\left(Y^{*},\left(y_{i}^{*}, x_{i}^{*}\right)_{i \in N}\right)$ is attained at the subgame-perfect Nash equilibrium

$$
Y^{*}=\sum_{i \in N} y_{i}^{*}, y_{1}^{*}=0, y_{2}^{*}=\frac{w_{2}}{1+p_{2}}, x_{1}^{*}=w_{1}, \text { and } x_{2}^{*}=\frac{w_{2}}{1+p_{2}} .
$$

While the payoff to agent 2 is $Y^{*}$, that to agent 1 is $w_{1}$, which is smaller than $Y^{*}$. To achieve the s-Pareto-efficient allocation through the transfer, by Proposition 6 agent 2 must transfer in such a way that agent 1 is the only contributor. Agent 1 is the only contributor if and only if the transfer from agent 2 to agent 1 is at least $\left(\left(1+p_{1}\right) w_{2}-w_{1}\right) /\left(2+p_{1}\right)>0 .^{7}$ Within the range of the transfer, the maximum payoff to agent 2 is $\left(w_{1}+w_{2}\right) /\left(2+p_{1}\right)$, which can be obtained when he/she sets $t_{21}=\left(\left(1+p_{1}\right) w_{2}-w_{1}\right) /\left(2+p_{1}\right)$. If $\left(w_{1}+w_{2}\right) /\left(2+p_{1}\right)$ is smaller than $Y^{*}$, he/she does not transfer $t_{21}$ : he/she does not transfer if

$$
\frac{w_{1}+w_{2}}{2+p_{1}}<\frac{w_{2}}{1+p_{2}} \text { or } w_{1}<\frac{1+p_{1}-p_{2}}{1+p_{2}} w_{2}
$$

\footnotetext{
${ }^{7}$ The ex post endowment of the private good of agent 1 is $w_{1}^{\prime}=w_{1}+t_{21}$ and that of agent 2 is $w_{2}^{\prime}=w_{2}-t_{21}$. Agent 1 is the only contributor after the transfer only if $w_{2}^{\prime} \leq w_{1}^{\prime} /\left(1+p_{1}\right)$. That is, $t_{21} \geq\left(\left(1+p_{1}\right) w_{2}-w_{1}\right) /\left(2+p_{1}\right)$. Note also that $w_{2}>\left(1+p_{2}\right) w_{1}$ implies $\left(1+p_{1}\right) w_{2}>w_{1}$.
} 
Note that the conditions $p_{1}<p_{2}$, and $\left(1+p_{2}\right) w_{1}<w_{2}, w_{1}<\left(1+p_{1}-p_{2}\right) w_{2} /(1+$ $\left.p_{2}\right)$ are compatible; for example, $\left(p_{1}, p_{2}, w_{1}, w_{2}\right)=(0.1,1,0.5,20)$ satisfies all conditions. Therefore, under the conditions, it is impossible to attain the s-Pareto-efficient allocation even if agents can freely transfer the private good to each other. Furthermore, note that the parameter values do not satisfy (9). However, Pareto improvement from $\left(Y^{*},\left(y_{i}^{*}, x_{i}^{*}\right)_{i \in N}\right)$ is possible because we can find an allocation that Pareto dominates $\left(Y^{*},\left(y_{i}^{*}, x_{i}^{*}\right)_{i \in N}\right)$ by applying (8). Such a Pareto improvement can successfully be executed if there is coercion to do so.

Second, (9) includes interesting cases. The first case is that every agent has the same initial endowment of the private good. In this case, the voluntary endowment transfer of the private good achieves a Pareto-efficient allocation because (9) holds in this situation.

Corollary 1 Suppose that $w_{i}=w>0$ for each $i \in N$. An s-Pareto-efficient allocation can be achieved at a subgame-perfect Nash equilibrium of the voluntary participation game with side payments.

The second case is more general than the first. This is a case in which every agent is a contributor (without transfers). We can show that (9) implies that every agent contributes to a positive level of the public good. From equation (4), we have the condition under which every agent contributes to a positive amount of a public good

$$
y_{i, N}^{*}=w_{i}+w_{i} \sum_{h \in N} \frac{1}{p_{h}}-\sum_{h \in N} \frac{w_{h}}{p_{h}}=\left(1+\sum_{h \in N \backslash\{i\}} \frac{1}{p_{h}}\right) w_{i}-\sum_{h \in N \backslash\{i\}} \frac{w_{h}}{p_{h}}>0 \text { for any } i .
$$

By Proposition 1, if $y_{j, N}^{*}>0$ such that $j \in \arg \min _{k \in N} w_{k}$, then $y_{i, N}^{*}>0$ for each $i \in N \backslash\{j\}$. Differentiating $y_{j, N}^{*}$ with respect to $p_{i}(i \neq 1)$, we have $\left(w_{i}-w_{j}\right) / p_{i}^{2}$. This is nonnegative because $w_{j}$ has the lowest value among $w_{k}$. This means that $y_{j, N}^{*}$ does not increase even if we replace $p_{i}$ by $p_{1}$. For all $p_{i}$ such that $i \neq 1$, we replace $p_{i}$ by $p_{1}$ and 
then the condition becomes

$$
y_{j, N}^{*} \geq\left(1+\frac{n-1}{p_{1}}\right) w_{j}-\sum_{h \in N \backslash\{j\}} \frac{w_{h}}{p_{1}}=\frac{1}{p_{1}}\left(\left(n+p_{1}\right) w_{j}-\sum_{h \in N} w_{h}\right)>0 .
$$

This is equivalent to the left-hand side of (9) in Proposition 7.

\section{Discussion}

One may conjecture that the strong complementarity between the private and public good strengthens the incentive for voluntary contribution to the public good. We discuss how strong complementarity affects the incentive to contribute voluntarily to the public good by comparing our results with those of Saijo and Yamato $(1999,2010)$.

We introduce the notion of group efficiency by naturally extending the i-Pareto efficiency in Definition 1: let $P \subseteq N$ be a set of participants. (i) An allocation $\left(Y,\left(y_{i}, x_{i}\right)_{i \in P}\right)$ is individually feasible for $P$ if $w_{i} \geq p_{i} y_{i}+x_{i}$ for each $i \in P$ and $Y=\sum_{i \in P} y_{i}$. (ii) An allocation for $P,\left(Y,\left(y_{i}, x_{i}\right)_{i \in P}\right)$, is group efficient within the set of individually feasible allocations for $P$ - henceforth, i-group efficient for $P$ for short - if there is no individually feasible allocation for $P,\left(Y^{\prime},\left(y_{i}^{\prime}, x_{i}^{\prime}\right)_{i \in P}\right)$, such that $U_{i}\left(Y^{\prime}, x_{i}^{\prime}\right) \geq U_{i}\left(Y, x_{i}\right)$ for all $i \in P$ with strict inequality for at least one $i \in P$. For each set of participants, the second stage of the voluntary participation game achieves i-group efficiency at a Nash equilibrium if every agent has the same Leontief utility function.

Proposition 8 Suppose that every agent has the same Leontief utility function. For each $P \subseteq N$, the voluntary contribution game when $P$ is the set of agents achieves i-groupefficient allocation for $P$ at a Nash equilibrium.

We can illustrate Proposition 8 in a similar way to Proposition 5. At the Nash equilibrium of the voluntary contribution game, $\sum_{j \in C\left(P, y_{P}^{*}\right)} y_{j, P}^{*}=w_{i}-p_{i} y_{i, P}^{*}$ for each $i \in C\left(P, y_{P}^{*}\right)$ and $\sum_{j \in C\left(P, y_{P}^{*}\right)} y_{j, P}^{*} \geq w_{i}$ for each $i \in P \backslash C\left(P, y_{P}^{*}\right)$. No $i \in P \backslash C\left(P, y_{P}^{*}\right)$ can be made better off because $w_{i}$ is the maximum payoff that $i$ can obtain. For $i \in C\left(P, y_{P}^{*}\right)$ 
to be made better off, other agents $j \in P \backslash\{i\}$ must increase their contribution to the public good, which reduces $j$ 's payoff.

Saijo and Yamato $(1999,2010)$ study the participation problem in the voluntary contribution game. They use the same model as we do, except for agents' utility functions. In their model, agents have the Cobb-Douglas utility function and quasilinear function, which have weaker complementarity between the private and the public goods than the Leontief utility function. In their case, the equilibrium allocation of the voluntary contribution game does not satisfy i-group efficiency for a set of participants, in particular the set consisting of more than two participants. Saijo and Yamato (1999, 2010) show that not all agents participate in the voluntary contribution to the public good and the equilibrium allocation of the participation game is not i-Pareto efficient.

Their results are in contrast to ours. In the presence of the strong complementarity modeled by the Leontief utility function, for each set of participants, the i-group-efficient allocation is supported at a Nash equilibrium by Proposition 8 and the i-Pareto-efficient allocation is attained at a subgame-perfect Nash equilibrium of the participation game by Proposition 5. From the comparison, we can conclude that when the private good is not transferable, the strong complementarity between the two goods provides such a strong incentive to agents as to achieve the (i-)Pareto-efficient allocation at equilibria.

When the private good is transferable, the implication of our result is the same as that of Saijo and Yamato (1999, 2010). As discussed in Section 4, the equilibrium allocation is not s-Pareto efficient in the voluntary participation game without transfer, even in our model. Strong complementarity cannot provide an incentive such that the equilibrium allocation is (s-)Pareto efficient. In our model, there is the possibility that $C\left(N, y_{N}^{*}\right)$ consists of many agents. Then, many agents participate in the equilibrium. The participation of many agents does not induce the (s-)Pareto efficiency of the equilibrium allocation. 


\section{Conclusion}

This paper examines the participation behavior and the allocative efficiency in the economy with a public good in the presence of the strong complementarity between the private good and the public good. In our model, every agent has the same Leontief utility function with respect to the consumption of the private and the public goods. Agents can differ in initial endowments of the private good and in production technology of the public good. We first find that whether agents voluntarily participate in the public good provision depends solely on the initial endowment of the private good: the greater the initial endowment of the private good that agents have, the more likely they are to participate in the provision of the public good. Such a binary participation decision does not depend on the efficiency of the production technology held by agents. Second, we examine the efficiency of the equilibrium allocation of the voluntary participation game. We show that while the equilibrium allocation is i-Pareto efficient, it is not necessarily s-Pareto efficient. Third, we examine whether the voluntary transfer of the private good among agents can achieve an s-Pareto-efficient allocation. We extend the voluntary participation game in such a way that agents can freely exchange their endowments of the private good, based on the model of Jackson and Wilkie (2005). We show that at the equilibrium in this model, the voluntary transfer scheme does not always achieve s-Pareto efficiency. The reason that the transfer scheme does not work is that the Pareto-improving transfer is always possible, but in some cases, it is difficult to provide agents with an incentive to execute such a transfer scheme. In this case, an outside authority such as a government is needed to compel a Pareto-improving transfer scheme.

Our result is new in the sense that we explain the incentive for the voluntary development of OSS in terms of the amount of endowment/resources a developer can invest in OSS. Our result can be partially justified by the empirical analysis by Bitzer and Geishecker (2010). We hope that our results will provide a new perspective on the study 
of the voluntary development of OSS.

\section{Appendix}

\section{Proof of Lemma 4}

(4.1) Consider a voluntary contribution game when $P \cup\{i\}$ is a set of participants. Suppose, conversely, that $y_{i, P \cup\{i\}}^{*}=0$. Then, $\sum_{j \in P \cup\{i\}} y_{j, P \cup\{i\}}^{*} \geq w_{i}$. Consider the game in which given $y_{i, P \cup\{i\}}^{*}=0$, agents in $P$ decide on their contributions, simultaneously. This is the same situation as in the game when $P$ is the set of participants. Thus, $C\left(P \cup\{i\}, y_{P \cup\{i\}}^{*}\right)=$ $C\left(P, y_{P}^{*}\right)$ and $w_{i}>\sum_{j \in P \cup\{i\}} y_{j, P \cup\{i\}}^{*}=\sum_{j \in P} y_{j, P}^{*}$. This is a contradiction.

(4.2) Let us denote $\mathcal{B} \equiv C\left(P \cup\{i\}, y_{P \cup\{i\}}^{*}\right) \cap C\left(P, y_{P}^{*}\right), \mathcal{C} \equiv C\left(P, y_{P}^{*}\right) \backslash C\left(P \cup\{i\}, y_{P \cup\{i\}}^{*}\right)$, and $\mathcal{D} \equiv C\left(P \cup\{i\}, y_{P \cup\{i\}}^{*}\right) \backslash C\left(P, y_{P}^{*}\right)$. By Lemma $4, i \in \mathcal{D}$; hence, $\mathcal{D}$ is not empty.

Claim 1 It follows that

$$
\sum_{l \in \mathcal{C}} \frac{w_{l}}{p_{l}}\left(1+\sum_{k \in \mathcal{B}} \frac{1}{p_{k}}+\sum_{k \in \mathcal{D}} \frac{1}{p_{k}}\right) \leq \sum_{l \in \mathcal{C}} \frac{1}{p_{l}}\left(\sum_{k \in \mathcal{B}} \frac{w_{k}}{p_{k}}+\sum_{k \in \mathcal{D}} \frac{w_{k}}{p_{k}}\right)
$$

and

$$
\sum_{k \in \mathcal{D}} \frac{w_{k}}{p_{k}}\left(1+\sum_{k \in \mathcal{B}} \frac{1}{p_{k}}\right)>\sum_{k \in \mathcal{D}} \frac{1}{p_{k}} \sum_{k \in \mathcal{B}} \frac{w_{k}}{p_{k}} .
$$

Proof of Claim 1. We first show (11). Because $y_{l, P \cup\{i\}}^{*}=0$ for each $l \in \mathcal{C}$, we have

$$
w_{l} \leq \frac{\sum_{k \in C\left(P \cup\{i\}, y_{P \cup\{i\}}^{*}\right)} \frac{w_{k}}{p_{k}}}{1+\sum_{k \in C\left(P \cup\{i\}, y_{P \cup\{i\}}^{*}\right)} \frac{1}{p_{k}}}=\frac{\sum_{k \in \mathcal{B}} \frac{w_{k}}{p_{k}}+\sum_{k \in \mathcal{D}} \frac{w_{k}}{p_{k}}}{1+\sum_{k \in \mathcal{B}} \frac{1}{p_{k}}+\sum_{k \in \mathcal{D}} \frac{1}{p_{k}}}
$$

for each $l \in \mathcal{C}$. By this condition

$$
\frac{w_{l}}{p_{l}} \leq \frac{\sum_{k \in \mathcal{B}} \frac{w_{k}}{p_{k}}+\sum_{k \in \mathcal{D}} \frac{w_{k}}{p_{k}}}{p_{l}\left(1+\sum_{k \in \mathcal{B}} \frac{1}{p_{k}}+\sum_{k \in \mathcal{D}} \frac{1}{p_{k}}\right)}
$$

for each $l \in \mathcal{C}$. Summarizing this condition over $l \in \mathcal{C}$ yields

$$
\sum_{l \in \mathcal{C}} \frac{w_{l}}{p_{l}} \leq \sum_{l \in \mathcal{C}} \frac{1}{p_{l}}\left(\frac{\sum_{k \in \mathcal{B}} \frac{w_{k}}{p_{k}}+\sum_{k \in \mathcal{D}} \frac{w_{k}}{p_{k}}}{1+\sum_{k \in \mathcal{B}} \frac{1}{p_{k}}+\sum_{k \in \mathcal{D}} \frac{1}{p_{k}}}\right)
$$


or

$$
\sum_{l \in \mathcal{C}} \frac{w_{l}}{p_{l}}\left(1+\sum_{k \in \mathcal{B}} \frac{1}{p_{k}}+\sum_{k \in \mathcal{D}} \frac{1}{p_{k}}\right) \leq \sum_{l \in \mathcal{C}} \frac{1}{p_{l}}\left(\sum_{k \in \mathcal{B}} \frac{w_{k}}{p_{k}}+\sum_{k \in \mathcal{D}} \frac{w_{k}}{p_{k}}\right) .
$$

Thus, we have (11).

Second, we show (12). When $P \cup\{i\}$ is a set of participants, every $l \in \mathcal{D}$ is a contributor; hence

$$
w_{l}>\frac{\sum_{k \in \mathcal{B}} \frac{w_{k}}{p_{k}}+\sum_{k \in \mathcal{D}} \frac{w_{k}}{p_{k}}}{1+\sum_{k \in \mathcal{B}} \frac{1}{p_{k}}+\sum_{k \in \mathcal{D}} \frac{1}{p_{k}}}
$$

for each $l \in \mathcal{D}$. Dividing this condition by $p_{l}$ for each $l \in \mathcal{D}$, we obtain

$$
\frac{w_{l}}{p_{l}}>\frac{\sum_{k \in \mathcal{B}} \frac{w_{k}}{p_{k}}+\sum_{k \in \mathcal{D}} \frac{w_{k}}{p_{k}}}{p_{l}\left(1+\sum_{k \in \mathcal{B}} \frac{1}{p_{k}}+\sum_{k \in \mathcal{D}} \frac{1}{p_{k}}\right)} .
$$

Summarizing this condition over $i \in \mathcal{D}$, we have

$$
\sum_{k \in \mathcal{D}} \frac{w_{k}}{p_{k}}>\sum_{k \in \mathcal{D}} \frac{1}{p_{k}}\left(\frac{\sum_{k \in \mathcal{B}} \frac{w_{k}}{p_{k}}+\sum_{k \in \mathcal{D}} \frac{w_{k}}{p_{k}}}{1+\sum_{k \in \mathcal{B}} \frac{1}{p_{k}}+\sum_{k \in \mathcal{D}} \frac{1}{p_{k}}}\right)
$$

or

$$
\sum_{k \in \mathcal{D}} \frac{w_{k}}{p_{k}}\left(1+\sum_{k \in \mathcal{B}} \frac{1}{p_{k}}+\sum_{k \in \mathcal{D}} \frac{1}{p_{k}}\right)>\sum_{k \in \mathcal{D}} \frac{1}{p_{k}}\left(\sum_{k \in \mathcal{B}} \frac{w_{k}}{p_{k}}+\sum_{k \in \mathcal{D}} \frac{w_{k}}{p_{k}}\right) .
$$

This is simplified as

$$
\sum_{k \in \mathcal{D}} \frac{w_{k}}{p_{k}}\left(1+\sum_{k \in \mathcal{B}} \frac{1}{p_{k}}\right)>\sum_{k \in \mathcal{D}} \frac{1}{p_{k}} \sum_{k \in \mathcal{B}} \frac{w_{k}}{p_{k}} .
$$

Hence, (12) holds.

(End of Proof of Claim 1)

The difference between $\sum_{k \in P \cup\{i\}} y_{k, P \cup\{i\}}^{*}$ and $\sum_{k \in P} y_{k, P}^{*}$ is

$$
\begin{aligned}
& \sum_{k \in P \cup\{i\}} y_{k, P \cup\{i\}}^{*}-\sum_{k \in P} y_{k, P}^{*} \\
= & \frac{1}{\left(1+\sum_{k \in C\left(P \cup\{i\}, y_{P \cup\{i\}}^{*}\right)} \frac{1}{p_{k}}\right)\left(1+\sum_{k \in C\left(P, y_{P}^{*}\right)} \frac{1}{p_{k}}\right)} \\
\times & \underbrace{\left[\sum_{k} \frac{w_{k}}{p_{k}}\left(1+\sum_{k \in C\left(P, y_{P}^{*}\right)} \frac{1}{p_{k}}\right)-\sum_{k \in C\left(P, y_{P}^{*}\right)} \frac{w_{k}}{p_{k}}\left(1+\sum_{k \in C\left(P \cup\{i\}, y_{P \cup\{i\}}^{*}\right)} \frac{1}{p_{k}}\right)\right]}_{\left(\alpha \in C\left(P \cup\{i\}, y_{P \cup\{i\}}^{*}\right)\right.} .
\end{aligned}
$$


Note that $C\left(P, y_{P}^{*}\right)=\mathcal{B} \cup \mathcal{C}$ and $C\left(P \cup\{i\}, y_{P \cup\{i\}}^{*}\right)=\mathcal{B} \cup \mathcal{D}$. Thus

$$
\begin{aligned}
(\alpha) & =\left(\sum_{k \in \mathcal{B}} \frac{w_{k}}{p_{k}}+\sum_{k \in \mathcal{D}} \frac{w_{k}}{p_{k}}\right)\left(1+\sum_{k \in \mathcal{B}} \frac{1}{p_{k}}+\sum_{k \in \mathcal{C}} \frac{1}{p_{k}}\right)-\left(\sum_{k \in \mathcal{B}} \frac{w_{k}}{p_{k}}+\sum_{k \in \mathcal{C}} \frac{w_{k}}{p_{k}}\right)\left(1+\sum_{k \in \mathcal{B}} \frac{1}{p_{k}}+\sum_{k \in \mathcal{D}} \frac{1}{p_{k}}\right) \\
& =\left(\sum_{k \in \mathcal{B}} \frac{w_{k}}{p_{k}}\right)\left(\sum_{k \in \mathcal{C}} \frac{1}{p_{k}}\right)+\sum_{k \in \mathcal{D}} \frac{w_{k}}{p_{k}}\left(1+\sum_{k \in \mathcal{B}} \frac{1}{p_{k}}+\sum_{k \in \mathcal{C}} \frac{1}{p_{k}}\right) \\
& -\left(\sum_{k \in \mathcal{B}} \frac{w_{k}}{p_{k}}\right)\left(\sum_{k \in \mathcal{D}} \frac{1}{p_{k}}\right)-\sum_{k \in \mathcal{C}} \frac{w_{k}}{p_{k}}\left(1+\sum_{k \in \mathcal{B}} \frac{1}{p_{k}}+\sum_{k \in \mathcal{D}} \frac{1}{p_{k}}\right) .
\end{aligned}
$$

By (11)

$$
\begin{aligned}
(\alpha) & \geq\left(\sum_{k \in \mathcal{B}} \frac{w_{k}}{p_{k}}\right)\left(\sum_{k \in \mathcal{C}} \frac{1}{p_{k}}\right)+\sum_{k \in \mathcal{D}} \frac{w_{k}}{p_{k}}\left(1+\sum_{k \in \mathcal{B}} \frac{1}{p_{k}}+\sum_{k \in \mathcal{C}} \frac{1}{p_{k}}\right) \\
& -\left(\sum_{k \in \mathcal{B}} \frac{w_{k}}{p_{k}}\right)\left(\sum_{k \in \mathcal{D}} \frac{1}{p_{k}}\right)-\sum_{k \in \mathcal{C}} \frac{1}{p_{k}}\left(\sum_{k \in \mathcal{B}} \frac{w_{k}}{p_{k}}+\sum_{k \in \mathcal{D}} \frac{w_{k}}{p_{k}}\right) . \\
& =\sum_{k \in \mathcal{D}} \frac{w_{k}}{p_{k}}\left(1+\sum_{k \in \mathcal{B}} \frac{1}{p_{k}}\right)-\left(\sum_{k \in \mathcal{B}} \frac{w_{k}}{p_{k}}\right)\left(\sum_{k \in \mathcal{D}} \frac{1}{p_{k}}\right) .
\end{aligned}
$$

By (12), we find that the left-hand-side value is larger than the right-hand-side one. That is, we have $(\alpha)>0$. Thus, $\sum_{k \in P \cup\{i\}} y_{k, P \cup\{i\}}^{*}>\sum_{k \in P} y_{k, P}^{*}$.

(4.3) By (4.1), $C\left(P \cup\{i\}, y_{P \cup\{i\}}^{*}\right) \cap C\left(P, y_{P}^{*}\right) \cup\{i\} \subseteq C\left(P \cup\{i\}, y_{P \cup\{i\}}^{*}\right)$. We show the converse. Let $j \in C\left(P \cup\{i\}, y_{P \cup\{i\}}^{*}\right)$. If $j=i$, then $j \in C\left(P \cup\{i\}, y_{P \cup\{i\}}^{*}\right) \cap C\left(P, y_{P}^{*}\right) \cup\{i\}$, trivially. We need to show that if $j \neq i$, then $j \in C\left(P, y_{P}^{*}\right)$. Suppose, conversely, that $j \notin C\left(P, y_{P}^{*}\right)$. Then, because $j \in C\left(P \cup\{i\}, y_{P \cup\{i\}}^{*}\right) \backslash C\left(P, y_{P}^{*}\right)$

$$
\sum_{k \in P \cup\{i\}} y_{k, P \cup\{i\}}^{*}=\frac{\left.\sum_{k \in C\left(P \cup\{i\}, y_{P \cup\{i\}}^{*}\right)}\right)^{\frac{w_{k}}{p_{k}}}}{1+\sum_{k \in C\left(P \cup\{i\}, y_{P \cup\{i\}}^{*}\right)} \frac{1}{p_{k}}}<w_{j} \leq \frac{\sum_{k \in C\left(P, y_{P}^{*}\right)} \frac{w_{k}}{p_{k}}}{1+\sum_{k \in C\left(P, y_{P}^{*}\right)} \frac{1}{p_{k}}}=\sum_{k \in P} y_{k, P}^{*},
$$

which contradicts (4.2).

\section{Proof of Lemma 5 .}

We show the contrapositive. Let $P \subsetneq N$ and $i \in N \backslash P$. Suppose that $i \in C\left(P \cup\{i\}, y_{P \cup\{i\}}^{*}\right)$ or $C\left(P \cup\{i\}, y_{P \cup\{i\}}^{*}\right) \neq C\left(P, y_{P}^{*}\right)$. Suppose first that $i \notin C\left(P \cup\{i\}, y_{P \cup\{i\}}^{*}\right)$ and $C(P \cup$ $\left.\{i\}, y_{P \cup\{i\}}^{*}\right) \neq C\left(P, y_{P}^{*}\right)$. Because $i \notin C\left(P \cup\{i\}, y_{P \cup\{i\}}^{*}\right)$, then $y_{i, P \cup\{i\}}^{*}=0$. Given $y_{i, P \cup\{i\}}^{*}=$ 
0, if agents in $P$ simultaneously choose their contributions, then they choose $y_{j, P}^{*}$ for all $j \in P$. Therefore, we must have $C\left(P \cup\{i\}, y_{P \cup\{i\}}^{*}\right)=C\left(P, y_{P}^{*}\right)$, which is a contradiction. Such a case never occurs. Second, suppose that $i \in C\left(P \cup\{i\}, y_{P \cup\{i\}}^{*}\right)$. By (4), if $i \in$ $C\left(P \cup\{i\}, y_{P \cup\{i\}}^{*}\right)$, then

$y_{i, P \cup\{i\}}^{*}=\frac{1}{p_{i}\left(1+\sum_{j \in C\left(P, y_{P}^{*}\right)}\left(1 / p_{j}\right)\right)}\left(w_{i}\left(1+\sum_{j \in C\left(P, y_{P}^{*}\right) \backslash\{i\}} \frac{1}{p_{j}}\right)-\sum_{j \in C\left(P, y_{P}^{*}\right) \backslash\{i\}} \frac{w_{j}}{p_{j}}\right)>0$.

By (iii) of Lemma $1, \sum_{j \in P \cup\{i\}} y_{j, P \cup\{i\}}^{*}=\left(1+\sum_{j \in C\left(P, y_{P}^{*}\right) \backslash\{i\}}\left(1 / p_{j}\right)\right) / \sum_{j \in C\left(P, y_{P}^{*}\right) \backslash\{i\}}\left(w_{j} / p_{j}\right)$. Thus, $w_{i}>\sum_{j \in P \cup\{i\}} y_{j, P \cup\{i\}}^{*}$.

\section{Proof of Proposition 4}

For preparation of proof of Proposition 4, we show the following claim.

Claim 2 Sets $N$ and $C\left(N, y_{N}^{*}\right)$ are Nash equilibrium sets of participants in the voluntary participation game.

Proof of Claim 2. We first show that $N$ is a Nash equilibrium set of participants. Suppose that $w_{i} \leq \sum_{j \in N \backslash\{i\}} y_{j, N \backslash\{i\}}^{*}$ for some $i \in C\left(N, y_{N}^{*}\right)$. Then, by Lemma $5, i \notin C\left(N, y_{N}^{*}\right)$. Thus, $w_{i}>\sum_{j \in N \backslash\{i\}} y_{j, N \backslash\{i\}}^{*}$ for each $i \in C\left(N, y_{N}^{*}\right)$. By Proposition $3, N$ is supported at a Nash equilibrium. Next, we show that $C\left(N, y_{N}^{*}\right)$ is a Nash equilibrium set of participants. By Lemma 5, $w_{i}>\sum_{j \in C\left(N, y_{N}^{*}\right) \backslash\{i\}} y_{j, C\left(N, y_{N}^{*}\right) \backslash\{i\}}$ for each $i \in C\left(C\left(N, y_{N}^{*}\right), y_{C\left(N, y_{N}^{*}\right)}^{*}\right){ }^{8}$ By Lemma $3, w_{k} \leq \sum_{j \in N} y_{j, N}^{*}=\sum_{j \in C\left(N, y_{N}^{*}\right)} y_{j, C\left(N, y_{N}^{*}\right)}^{*}$ for each $k \in N \backslash C\left(N, y_{N}^{*}\right)$. By Proposition $3, C\left(N, y_{N}^{*}\right)$ is supported at a Nash equilibrium. (End of Proof of Claim 2)

(sufficiency) By Lemma $5, w_{i}>\sum_{j \in P \backslash\{i\}} y_{j, P \backslash\{i\}}^{*}$ for each $i \in C\left(P, y_{P}^{*}\right)$. Before proving that $P$ satisfies (ii') in Proposition 3, we show that $C\left(N, y_{N}^{*}\right)=C\left(P, y_{P}^{*}\right)$. We show this by applying Lemma 5 iteratively to each agent in $P \backslash C\left(N, y_{N}^{*}\right)$. Denote $P \backslash C\left(N, y_{N}^{*}\right) \equiv$ $\left\{k_{1}, \ldots, k_{m}\right\}$ such that $m \geq 1 .^{9}$ Because $C\left(N, y_{N}^{*}\right) \subseteq P \subseteq N$, then $P \backslash C\left(N, y_{N}^{*}\right) \subseteq$

\footnotetext{
${ }^{8}$ Trivially, $C\left(C\left(N, y_{N}^{*}\right), y_{C\left(N, y_{N}^{*}\right)}^{*}\right)=C\left(N, y_{N}^{*}\right)$.

${ }^{9}$ If $P \backslash C\left(N, y_{N}^{*}\right)$ is empty, $C\left(N, y_{N}^{*}\right)=C\left(P, y_{P}^{*}\right)$ trivially holds.
} 
$N \backslash C\left(N, y_{N}^{*}\right)$. Because by Claim 2, $C\left(N, y_{N}^{*}\right)$ is supported at a Nash equilibrium, then

$$
w_{j} \leq \sum_{j \in C\left(N, y_{N}^{*}\right)} y_{j, C\left(N, y_{N}^{*}\right)}^{*} \text { for each } j \in N \backslash C\left(N, y_{N}^{*}\right)
$$

Hence, in particular, $w_{j} \leq \sum_{j \in C\left(N, y_{N}^{*}\right)} y_{j, C\left(N, y_{N}^{*}\right)}^{*}$ for each $j \in P \backslash C\left(N, y_{N}^{*}\right)$. First, we apply Lemma 5 to $k_{1}$. Note that $C\left(C\left(N, y_{N}^{*}\right), y_{C\left(N, y_{N}^{*}\right)}^{*}\right)=C\left(N, y_{N}^{*}\right)$. Because $w_{k_{1}} \leq$ $\sum_{j \in C\left(N, y_{N}^{*}\right)} y_{j, C\left(N, y_{N}^{*}\right)}^{*}$, then by Lemma $5, k_{1} \notin C\left(C\left(N, y_{N}^{*}\right) \cup\left\{k_{1}\right\}, y_{C\left(N, y_{N}^{*}\right) \cup\left\{k_{1}\right\}}^{*}\right)$ and $C\left(N, y_{N}^{*}\right)=C\left(C\left(N, y_{N}^{*}\right), y_{C\left(N, y_{N}^{*}\right)}^{*}\right)=C\left(C\left(N, y_{N}^{*}\right) \cup\left\{k_{1}\right\}, y_{C\left(N, y_{N}^{*}\right) \cup\left\{k_{1}\right\}}^{*}\right)$. Thus

$$
\sum_{j \in C\left(N, y_{N}^{*}\right)} y_{j, C\left(N, y_{N}^{*}\right)}^{*}=\sum_{j \in C\left(N, y_{N}^{*}\right) \cup\left\{k_{1}\right\}} y_{j, C\left(N, y_{N}^{*}\right) \cup\left\{k_{1}\right\}}^{*}
$$

Second, we apply Lemma 5 to $k_{2}$. By (15)

$$
w_{k_{2}} \leq \sum_{j \in C\left(N, y_{N}^{*}\right)} y_{j, C\left(N, y_{N}^{*}\right)}^{*}=\sum_{j \in C\left(N, y_{N}^{*}\right) \cup\left\{k_{1}\right\}} y_{j, C\left(N, y_{N}^{*}\right) \cup\left\{k_{1}\right\}}^{*}
$$

Thus, by Lemma 5

$$
C\left(C\left(N, y_{N}^{*}\right) \cup\left\{k_{1}\right\}, y_{C\left(N, y_{N}^{*}\right) \cup\left\{k_{1}\right\}}^{*}\right)=C\left(C\left(N, y_{N}^{*}\right) \cup\left\{k_{1}, k_{2}\right\}, y_{C\left(N, y_{N}^{*}\right) \cup\left\{k_{1}, k_{2}\right\}}^{*}\right) .
$$

By the iterative application of Lemma 5 to $k_{3}, \ldots, k_{m}$, we have

$$
\begin{aligned}
C\left(N, y_{N}^{*}\right) & =C\left(C\left(N, y_{N}^{*}\right) \cup\left\{k_{1}\right\}, y_{C\left(N, y_{N}^{*}\right) \cup\left\{k_{1}\right\}}^{*}\right)=C\left(C\left(N, y_{N}^{*}\right) \cup\left\{k_{1}, k_{2}\right\}, y_{C\left(N, y_{N}^{*}\right) \cup\left\{k_{1}, k_{2}\right\}}^{*}\right) \\
& =\cdots=C\left(P \backslash\left\{k_{m}\right\}, y_{P \backslash\left\{k_{m}\right\}}^{*}\right)=C\left(P, y_{P}^{*}\right) .
\end{aligned}
$$

By (16) and Lemma 3

$$
\sum_{j \in C\left(N, y_{N}^{*}\right)} y_{j, C\left(N, y_{N}^{*}\right)}^{*}=\sum_{j \in C\left(P, y^{*}\right)} y_{j, C\left(P, y_{P}^{*}\right)}^{*}=\sum_{j \in P} y_{j, P}^{*}
$$

Conditions (14) and (17) and $N \backslash P \subseteq N \backslash C\left(N, y_{N}^{*}\right)$ imply that $w_{j} \leq \sum_{j \in C\left(N, y_{N}^{*}\right)} y_{j, C\left(N, y_{N}^{*}\right)}^{*}=$ $\sum_{j \in P} y_{j, P}^{*}$ for each $j \in N \backslash P$, which is (ii').

(necessity) It is trivial that $P \subseteq N$. We show that each Nash equilibrium set of participants $P$ satisfies $C\left(N, y_{N}^{*}\right) \subseteq P$. Suppose, conversely, that there exists a Nash 
equilibrium set of participants $P$ that does not satisfy it. Then, there is $i \in C\left(N, y_{N}^{*}\right) \backslash P$, which implies $\sum_{j \in C\left(N, y_{N}^{*}\right)} y_{j, C\left(N, y_{N}^{*}\right)}^{*}=\sum_{j \in N} y_{j, N}^{*}<w_{i} \leq \sum_{j \in P} y_{j, P}^{*}$ by Lemma 3 . When $N=P$, this inequality does not hold. We need to consider the case where $P \neq N$. Because $P$ is supported at a Nash equilibrium, then $\sum_{j \in P} y_{j, P}^{*} \geq w_{k}$ for each $k \in N \backslash P$. Applying Lemma 5 to each agent in $N \backslash P$ iteratively in a similar way to the above, we have $C\left(P, y_{P}^{*}\right)=C\left(P \cup\{j\}, y_{P \cup\{j\}}^{*}\right)=\cdots=C\left(N \backslash\{l\}, y_{N \backslash\{l\}}^{*}\right)=C\left(N, y_{N}^{*}\right)$, where $j, l \in N \backslash P$.By Lemma $3, \sum_{j \in N} y_{j}^{*}=\sum_{j \in C\left(N, y_{N}^{*}\right)} y_{j, C\left(N, y_{N}^{*}\right)}^{*}=\sum_{j \in C\left(P, y_{P}^{*}\right)} y_{j, C\left(P, y_{P}^{*}\right)}^{*}=\sum_{j \in P} y_{j, P}^{*}$, which is a contradiction.

\section{References}

[1] Bergstrom, T., Blume, L., Varian, H., 1986. On the private provision of public goods. Journal of Public Economics 29(1), 25-49.

[2] Bitzer, J., Geishecker, I., 2010. Who contributes voluntarily to OSS? An investigation among German IT employees. Research Policy 39(1), 165-172.

[3] Bitzer, J., Schrettl, W., Schröder, P.J.H., 2007. Intrinsic motivation in open source software development. Journal of Comparative Economics 35(1), 160-169.

[4] Bitzer, J., Schröder, P.J.H., 2005. Bug-fixing and code-writing: The private provision of open source software. Information Economics and Policy 17(3), 389-406.

[5] Franke, N., von Hippel, E., 2003. Satisfying heterogeneous user needs via innovation toolkits: The case of Apache security software. Research Policy 32(7), 1199-1215.

[6] Furusawa, T., Konishi, H., 2011. Contributing or free-riding? Voluntary participation in a public good economy. Theoretical Economics 6(2), 219-256.

[7] Healy, P.J., 2010. Equilibrium participation in public goods allocations. Review of Economic Design 14(1-2), 27-50. 
[8] Jackson, M.O., Wilkie, S., 2005. Endogenous games and mechanisms: Side payments among players. Review of Economic Studies 72(2), 543-556.

[9] Johnson, J.P., 2002. Open source software: private provision of a public good. Journal of Economics and Management Strategy 11(4), 637-662.

[10] Konishi, H., Shinohara, R., 2012. Voluntary participation and the provision of public goods in large finite economies. Forthcoming in Journal of Public Economic Theory.

[11] Lakhani, K.R., von Hippel, E., 2003. How open source software works: "Free" userto-user assistance. Research Policy 32(6), 923-943.

[12] Lerner, J., Tirole, J., 2002. Some simple economics of open source. Journal of Industrial Economics 50(2), 197-234.

[13] von Hippel, E., von Krogh, G., 2003. Open source software and the "privatecollective" innovation model: Issues for organization science. Organization Science 14(2), 209-223.

[14] Saijo, T., Yamato, T., 1999. A voluntary participation game with a non-excludable public good. Journal of Economic Theory 84(2), 227-242.

[15] Saijo, T., Yamato, T., 2010. Fundamental impossibility theorems on voluntary participation in the provision of non-excludable public goods. Review of Economic Design $14(1-2), 51-73$.

[16] Shinohara, R., 2009. The possibility of efficient provision of a public good in voluntary participation games. Social Choice and Welfare 32(3), 367-387.

[17] Shinohara, R., 2011. Participation and demand levels for a joint project. Mimeo, Shinshu University. 